\title{
Neurovascular Units: A New Cost Effective Model?
}

Stroke units have shown to be cost effective for the care of a range of stroke patients (Cochrane Collaboration 2015) and intensive care of acute conditions, such as subarachnoid hemorrhage, also seem to justify their costs. ${ }^{1}$ Now Appel et al describe a model hybrid neurovascular unit that treats both types of patients. ${ }^{2}$

Stroke units decrease short-term and long-term mortality rates in the entire stroke population as well as in subgroups. Stroke unit care also reduces the need for long-term hospitalization. This difference in favour of the stroke unit was independent of the patients' age, the extent of neurological deficit on admission and previous history. In subgroups where the general prognosis is fair or good (minor neurological deficits and less than 75 years of age), stroke unit care accelerated the process of rehabilitation, in groups with a poor general prognosis (major deficits and more than 75 years of age), the ultimate proportion of patients able to return home was enhanced by stroke unit care. It is concluded by many randomized trials that care in a stroke unit benefits greatly the majority of stroke patients and that such a unit should be designed to admit all acute stroke patients without preselection. $^{3}$

There is also a need to care for patients with other acute neurovascular conditions. Data suggest that high-intensity staffing patterns in the intensive care unit (ICU) are associated with cost savings and improved outcomes. Many studies in literature investigated the cost-effectiveness and clinical outcomes of high intensity ICU, physician staffing as recommended by The Leapfrog Group (a consortium of companies that purchase health care for their employees) and identified ways to overcome barriers to nationwide implementation of these standards. Hospitals that have implemented the Leapfrog initiative have demonstrated reductions in mortality, length of stay and cost savings. ${ }^{4}$

Although the exact mechanisms that explain this observation are still largely unknown, recent literature suggests that highintensity and the presence of a multidisciplinary team both play key roles. Other factors that may explain the association between high-intensity intensive care unit physician staffing and improved outcomes include rapid access to critical care by an experienced critical care provider and consistent implementation of protocols to deliver evidence-based critical care. ${ }^{3}$

The Toronto Western Hospital (TWH) is an acute care academic hospital and regional stroke center. Stroke patients were treated by a mobile stroke team and the patients were admitted either to a general medical ward or the neurology ward. Patients with other acute neurovascular conditions, such as subarachnoid hemorrhage, were admitted to neurosurgery. A 20 bed neurovascular unit (NVU) was created to admit both types of patients.

Using retrospective patient level data from two years prior and one year post NVU, Appel et al suggest an overall savings of CDN $\$ 450,000$. $^{2}$
This figure may not reflect the complexities created by the establishment of the NVU. The annual prorated number of visits decreases from the pre-NVU to post-NVU period (from 652.6 to 627.9) and there is a shift in case mix with more patients with acute ischemic stroke (annual prorated from 227.5 to 256.0) and fewer patients with subarachnoid hemorrhage (from 95.3 to 74.4) and transient ischemic attack (TIA - from 52.4 to 27.7). The reduction in TIA is attributable to the implementation of a TIA and minor stroke unit; but the remaining changes still make for an apples-to-oranges comparison of costs before and after the introduction of the NVU.

Taking a closer look at the service-specific outcomes, the authors observed a decrease in the cost of each service individually, with cost per visit decreasing from $\$ 12,558$ to $\$ 10,623$ for General Internal Medicine service, $\$ 20,018$ to $\$ 19,073$ for Neurology service and from $\$ 32,301$ to $\$ 31,006$ for Neurosurgery service patients which may be related to the introduction of the NVU but may be related to other hospital-wide cost pressures. The introduction of the NVU likely did cause a reduction in the use of the relatively low-cost General Internal Medicine service (from $29.4 \%$ to $4.8 \%$ of patients) and an increase in the use of the highercost Neurology service (from $20.3 \%$ to $49.2 \%$ of patients). This shift in the responsibility of care to the NVU may have resulted in improved patient outcomes, reduced mortality or morbidity, or improved quality of life after discharge but it probably did not save CDN $\$ 450,000$.

The authors observed an increase in the alternative level of care length of stay which is unlikely to be consequence of the NVU and which may have impacted their evaluation. Concurrently with the introduction of the NVU, all services discharging to rehabilitation facilities and nursing homes may have experienced increases in alternative level length of stay which increase hospital costs and are unlikely to provide benefits to patients. As the authors stated, ensuring timely discharge when patients are clinically ready for discharge is important for managing hospital resources and will almost certainly save money.

A first year experience is seldom typical. Often those who participate in a new enterprise are pioneers and keen. The eyes are on the new initiative and may encourage performances that are unsustainable for the long term. On the other hand, with experience, processes become smoother and more efficient and initial errors can be redressed. The authors need to continue their studies, not only because of these considerations, but because they have not yet evaluated their relationship with the decreased length of stay and potential cost savings to outcomes. Moreover, as the authors acknowledge, the establishment of a TIA and minor stroke unit next door is bound to affect the operations of the

Received May 14, 2015. Final Revisions Submitted June 2, 2015. 
NVU, probably favorably. However, further studies on clinical outcomes are essential, since outcomes are the ultimate measure of success in health care.

\section{Disclosures}

The authors do not have anything to disclose.

Antonia Nucera, Lauren Cipriano, Vladimir Hachinski Western University, London, Ontario, Canada Email:Vladimir.hachinski@lhsc.on.ca

\section{REFERENCES}

1. De Reuck JL. Stroke Units Improving the Outcome of Patients with a Cerebrovascular Event. Eur Neurol Rev. 2006;6:64-7.

2. Appel E, Hahn-Goldberg S, Chow E, Casaubon L, Abrams H. The operational and economic impact of a neurovascular unit in an acute care academic hospital. Can J Neurol Sci. 2015, (in press).

3. Strand T, Asplund K, Eriksson S, Hägg E, Lithner F, Wester P. Stroke unit care-who benefits? Comparisons with general medical care in relation to prognostic indicators on admission. Stroke 1986; $17: 377-81$.

4. Logani S, Green A, Gasperino J. Benefits of High-Intensity Intensive Care Unit Physician Staffing under the Affordable Care Act. Crit Care Res Pract. 2001;2011:170814. 